\title{
Iterative Equalization and Interference Alignment for Multiuser MIMO HetNets with Imperfect CSI
}

\author{
Daniel Castanheira, ${ }^{1}$ Jorge Aido, ${ }^{1}$ Roberto Magueta, ${ }^{1}$ Adão Silva, \\ Atilio Gameiro, ${ }^{1}$ and Rui Dinis ${ }^{2}$ \\ ${ }^{1}$ Instituto de Telecomunicações, University of Aveiro, 3810-193 Aveiro, Portugal \\ ${ }^{2}$ Instituto de Telecomunicações, Faculdade de Ciências e Tecnologia, Universidade Nova de Lisboa, 2829-516 Lisboa, Portugal \\ Correspondence should be addressed to Daniel Castanheira; dcastanheira@av.it.pt
}

Received 31 July 2015; Revised 23 October 2015; Accepted 8 December 2015

Academic Editor: Francesco Gringoli

Copyright ( 2015 Daniel Castanheira et al. This is an open access article distributed under the Creative Commons Attribution License, which permits unrestricted use, distribution, and reproduction in any medium, provided the original work is properly cited.

\begin{abstract}
In this paper we consider a scenario, where several small-cells work under the same coverage area and spectrum of a macrocell. The signals stemming from the small-cell (macrocell) users if not carefully dealt with will generate harmful interference into the macrocell (small-cell). To tackle this problem interference alignment and iterative equalization techniques are considered. By using IA all interference generated by the small-cell (macrocell) users is aligned along a low dimensional subspace, at the macrocell (small-cells). This reduces considerably the amount of resources allocated, to enable the coexistence of the two systems. However, perfect IA requires the availability of error-free channel state information (CSI) at the transmitters. Due to CSI errors one can have substantial performance degradation due to imperfect alignments. Since in this work the IA precoders are based on imperfect CSI, an efficient iterative space-frequency equalization is designed at the receiver side to cope with the residual aligned interference. The results demonstrate that iterative equalization is robust to imperfect CSI and removes efficiently the interference generated by the poorly aligned interference. Close to matched filter bound performance is achieved, with a very few number of iterations.
\end{abstract}

\section{Introduction}

The operators are considering the deployment of smaller cells (e.g., picocells and femtocells) along its macrocells to cope with the exponential demand for data traffic verified in the last years $[1,2]$. However, a higher network densification has also its limitations. More cells per unit area leads to higher levels of interference and as a consequence the system performance is degraded $[2,3]$. To deal with this interference, the small-cells must reuse the macrocell resources, since the macrocell is the owner of the spectrum.

The use of the space domain is of special importance when dealing with interference in an heterogeneous based network environment. Zero-forcing (ZF) is the most commonly used technique to deal with interference. ZF works by inserting a null in the direction of the terminal subject to interference. Interference alignment (IA) is another option to deal with this type of interference, especially when the interference is generated by several terminals. IA is a linear precoding technique that manages this type of interference by aligning the multiterminal interference along a low dimensional subspace, whereas the intended signal space spans a different subspace. For the interference channel, IA has been shown to achieve the degrees of freedom outer bound $[4,5]$. Closed-form solutions are only available for some specific cases [6], while for the general case IA approaches are based on iterative methods [6-8]. In [6], two iterative algorithms were proposed that take advantage of reciprocity in wireless networks to achieve IA, with only local channel knowledge at each node. A convergent alternating minimization approach to IA was proposed in [7]. In [8] the authors presented two algorithms that optimize the precoding subspaces by maximizing the data rate performance while maintaining the achievable degrees of freedom.

In the context of heterogeneous networks, the works in [9-12] studied some IA techniques to deal with the interference generated by the small-cell user terminals (UTs) towards the macrocell. In [9] the authors proposed a new 
IA scheme that successively creates transmit beamforming vectors for the small-cell terminals and for the macroBS assuming that they have different number of transmit antennas. The work in [10] studied IA techniques with different levels of intersystem information exchange. In [11] the spatial dimensions left available by the macrocell were used to transmit the small-cells data. In this work only a single macrocell UT is scheduled per subcarrier and perfect CSI is available at the transmitters. The interference generated by the small-cells is aligned in an orthogonal subspace to the macrocell received signal subspace. The case of IA precoder designed under limited information exchange has been analytically tackled in [13], for narrowband heterogeneous based networks.

Single-carrier frequency division multiple access (SCFDMA) has a lower peak-to-average power ratio (PAPR) than OFDMA [14]. As the mobile terminals are low power devices SC-FDMA has been adopted, for the uplink, as the multiple access scheme of the current long-term evolution (LTE) cellular system [13]. It is also a promising solution technique for high data rate uplink communications in future cellular systems. Single-carrier frequency domain equalization (SCFDE) is widely recognized as an excellent alternative to OFDM, especially for the uplink of broadband wireless systems $[15,16]$. The SC-FDE based schemes may be either linear or nonlinear. Optimized linear frequency domain equalization (FDE) is widely used in conventional SC-FDE schemes. However, it suffers from high residual interference levels, leading to poor performance, that is, several $\mathrm{dB}$ from the matched filter bound (MFB) [17]. On the other hand, nonlinear FDE even if more complex do not suffer from this drawback. For this reason, the designing of nonlinear FDE in general and decision-feedback FDE in particular, with the iterative block decision-feedback equalization (IB-DFE) being the most promising nonlinear FDE [17] has gained significant attention lately. IB-DFE was originally proposed in [18] and was extended for a wide range of scenarios in the last years, ranging between diversity scenarios, multiplexing MIMO, and MC-CDMA systems, among many others [1926].

Contributions. In this paper we design a practical and efficient multitier IA-precoding with iterative space-frequency equalization approach to cope with the residual intertier alignment interference, caused by the imperfect knowledge of the CSI at the terminals. The main contributions of the paper are as follows.

(i) We extend the work of [11] by considering a more general and realistic case, where the macrocell serves more than one user per subcarrier. The schemes proposed in [14], were designed by assuming that the macrocell only serves a single user. Therefore, we use IA to align the generated interference along two predefined subspaces (one for the macrocell and another for the small-cells). These subspaces are assumed to be known in advance at both cells. Furthermore, a generic number of antenna terminals are considered contrarily to [11], where the schemes were designed for a specific set of antennas. (ii) The proposed iterative equalizer scheme is designed considering explicitly imperfect CSI, contrarily to [14], where perfect CSI was assumed. Furthermore, as SC-FDMA terminals are also prone to intercarrier interference (ICI) the equalizer design takes into account not only the residual interference but also the ICI.

(iii) Additionally, a simple and accurate semianalytical approach is proposed to obtain the performance of the proposed receiver structure.

The results demonstrate that the proposed iterative algorithm achieves good performance with imperfect CSI, since it efficiently removes the residual intertier interference generated by the poor interference alignment. Moreover, the proposed schemes achieve a performance close to matched filter bound, with only 2-4 iterations. This means that with the proposed scheme the small-cell users can coexist under the same spectrum of the macrocell without significant interference, even under imperfect CSI knowledge, and with moderate complexity.

Organization. This paper is organized as follows. Section 2 presents the system model. In Section 3, we start by designing the IA precoder. Then the proposed receiver structures are presented in detail and a semianalytical approach for obtaining the performance is discussed. Section 4 presents the main simulation results. The conclusions will be drawn in Section 5.

Notation. Boldface capital letters denote matrices and boldface lowercase letters denote column vectors. The operations $(\cdot)^{H}$ and $\operatorname{tr}(\cdot)$ represent the Hermitian transpose and the trace of a matrix. $\left\{\alpha_{l}\right\}_{l=1}^{L}$ represents $L$ length sequence. $\mathbf{I}_{N}$ is the identity matrix with size $N \times N$. null(A) denotes a matrix whose columns span the null space of matrix $\mathbf{A} \cdot \mathbf{d}(p)$ and $\mathbf{D}(p)$ denote the element $p$ of vector $\mathbf{d}$ and the entry $(p, p)$ of diagonal matrix $\mathbf{D}$, respectively. The indexes $s$ and $m$ are used to represent the small-cell and the macrocell, respectively.

\section{System Model}

Let us consider $K_{s}$ small-cells working under the same spectrum and under the coverage area of a macrocell, as depicted in Figure 1. Both macro- and small-cell terminals use as access technique SC-FDMA with $L$ available subcarriers. The macrocell serves $K_{m}$ UT's and each small-cell serves one UT. In the following, the macrocell $k$ th user terminal is denominated by $\mathrm{UT}_{m, k}$ and the UT small-cell by $\mathrm{UT}_{s, k}$. The index $l$ is used to denote a given subcarrier of the $L$ available.

2.1. Macrocell System. At the macrocell a BS serves $K_{m}$ associated UTs, where the number of antennas at the BS and each UT is $M_{r}$ and $M_{t}$, respectively. The received frequency domain signal (i.e., after cyclic prefix removal and FFT operation) at the macrocell $\mathrm{BS}$ on the $l$ th subcarrier is given by

$$
\mathbf{y}_{m}^{l}=\underbrace{\mathbf{H}_{m m}^{l} \mathbf{x}_{m}^{l}}_{\text {Desired Signal }}+\underbrace{\mathbf{H}_{m s}^{l} \mathbf{x}_{s}^{l}}_{\begin{array}{c}
\text { Inter-System } \\
\text { Interference }
\end{array}}+\underbrace{\mathbf{n}_{m}^{l}}_{\text {Noise }},
$$




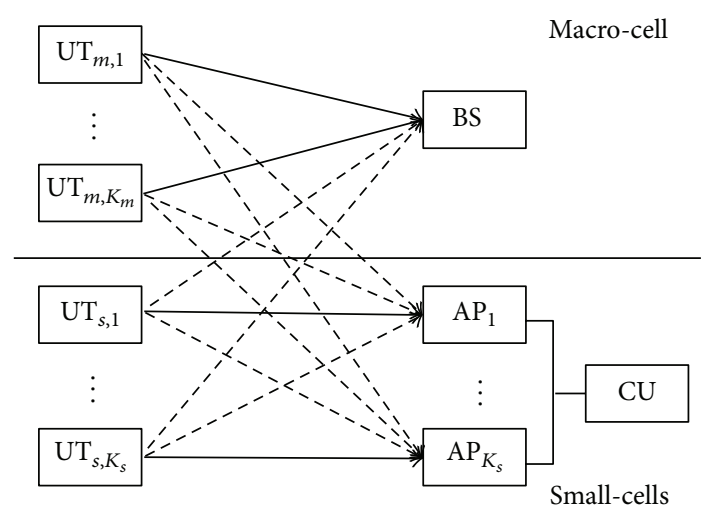

FIGURE 1: Block diagram of the considered heterogeneous system.

where $\mathbf{H}_{m m}^{l}$ and $\mathbf{H}_{m s}^{l}$ are block matrices; that is, $\mathbf{H}_{m m}^{l}=\left[\mathbf{H}_{m m, 1}^{l}, \ldots, \mathbf{H}_{m m, K_{m}}^{l}\right] \in \mathbb{C}^{M_{r} \times K_{m} M_{t}}$ and $\mathbf{H}_{m s}^{l}=\left[\mathbf{H}_{m s, 1}^{l}, \ldots, \mathbf{H}_{m s, K}^{l}\right] \in \mathbb{C}^{M_{r} \times N_{t} K_{s}} . \mathbf{H}_{m m, K}^{l}\left(\mathbf{H}_{m s, K}^{l}\right)$ denotes the channel matrix between $\mathrm{UT}_{m, K}\left(\mathrm{UT}_{s, K}\right)$ and the BS on the $l$ th subcarrier. $\mathbf{x}_{m}^{l}=\left[\mathbf{x}_{m, 1}^{H}, \ldots, \mathbf{x}_{m, K_{m}}^{H}\right]^{H} \in$ $\mathbb{C}^{S_{m} K_{m}}\left(\mathbf{x}_{s}^{l}=\left[\mathbf{x}_{s, 1}^{H}, \ldots, \mathbf{x}_{s, K_{s}}^{H}\right]^{H} \in \mathbb{C}^{S_{s} K_{s}}\right)$ represents the concatenation of all macrocell UTs (small-cells UT) transmit signals on the $l$ th subcarrier, and $\mathbf{n}_{m}^{l}$ white Gaussian noise with variance $\sigma_{\text {noise }}^{2} \cdot S_{m}$ denotes the number of streams sent by each macrocell terminal and $S_{s}$ denotes the number of streams sent by each small-cell terminal.

The macro- $\mathrm{UT}_{m, k}$ transmit signal is given by

$$
\begin{aligned}
\mathbf{x}_{m, k}^{l} & =\mathbf{V}_{m, k}^{l} \mathbf{z}_{m, k}^{l}, \\
\left\{\mathbf{z}_{m, k}^{l}(p)\right\}_{l=1}^{L} & =\operatorname{DFT}\left(\left\{\mathbf{d}_{m, k}^{l}(p)\right\}_{l=1}^{L}\right),
\end{aligned}
$$

where $\mathbf{z}_{m, k}^{l}(p)$ is the discrete Fourier transform (DFT) of the sequence $\left\{\mathbf{d}_{m, k}^{l}(p)\right\}_{l=1}^{L}, \mathbf{V}_{m, k}^{l} \in \mathbb{C}^{M_{t} \times S_{m}}$ is a unitary linear precoder $\left(\left(\mathbf{V}_{m, k}^{l}\right)^{H} \mathbf{V}_{m, k}^{l}=\mathbf{I}_{S_{m}}\right)$, and $\mathbf{d}_{m, k}^{l} \in \mathbb{C}^{M_{t}}$ denotes the vector of data symbols transmitted by $\mathrm{UT}_{m, k}$ at subcarrier $l$. The data symbols are drawn from a $M$-QAM constellation. $\mathbf{d}_{m, k}^{l}(p)$ is the $p$ element of $\mathbf{d}_{m, k}^{l}$ whose average power is constrained to $\mathbb{E}\left[\left|\mathbf{d}_{m, k}^{l}(p)\right|^{2}\right]=P_{m}$.

2.2. Small-Cells. Each small-cell has one access point (AP) and an associated UT. It is assumed that each AP and UT is equipped with $N_{r}$ and $N_{t}$ antennas, respectively. The received signal $\mathrm{y}_{s}^{l} \in \mathbb{C}^{N_{r} K_{s} \times 1}$ in frequency domain at the central unit, where the joint processing of all APs signals is done, and subcarrier $l$ is

$$
\mathbf{y}_{s}^{l}=\underbrace{\mathbf{H}_{s s}^{l} \mathbf{x}_{s}^{l}}_{\text {Desired Signal }}+\underbrace{\mathbf{H}_{s m}^{l} \mathbf{x}_{m}^{l}}_{\begin{array}{c}
\text { Inter-System } \\
\text { Interference }
\end{array}}+\underbrace{\mathbf{n}_{s}^{l}}_{\text {Noise }},
$$

where $\mathbf{H}_{s s}^{l} \in \mathbb{C}^{N_{r} K_{s} \times N_{t} K_{s}}$ and $\mathbf{H}_{s m}^{l} \in \mathbb{C}^{N_{r} K_{s} \times M_{t} K_{m}}$ denote the overall channels between the small-cell UTs to the APs and channel between the macrocell UTs to the APs on the $l$ th subcarrier, respectively. The matrix
$\mathbf{H}_{s s}^{l}=\left[\begin{array}{lllll}\mathbf{H}_{s s, 1}^{l} & \cdots & \mathbf{H}_{s s, n}^{l} & \cdots & \mathbf{H}_{s s, K_{s}}^{l}\end{array}\right]$, where $\mathbf{H}_{s s, n}^{l}=$ $\left[\begin{array}{lllll}\mathbf{H}_{s s, n, 1}^{l H} & \cdots & \mathbf{H}_{s s, n, i}^{l H} & \cdots & \mathbf{H}_{s s, n, K_{s}}^{l H}\end{array}\right]$ and $\mathbf{H}_{s s, n, i}^{l H}$ represents the channel matrix between the $n$th small-cell UT and the $i$ th AP. The same applies to channel matrix $\mathbf{H}_{s m}^{l} . \mathbf{n}_{s}^{l}$ is the zero mean white Gaussian noise with variance $\sigma_{\text {noise. }}^{2}$. The transmit signal at small-cell $\mathrm{UT}_{s, k}$ is obtained through the linear map $\mathbf{V}_{s, k}^{l}$ :

$$
\mathbf{x}_{s, k}^{l}=\mathbf{V}_{s, k}^{l} \mathbf{z}_{s, k}^{l} .
$$

The sequence $\left\{\mathbf{z}_{s, k}^{l}(p)\right\}_{l=1}^{L}$ is the DFT of the data symbols $\left\{\mathbf{d}_{s, k}^{l}(p)\right\}_{l=1}^{L}$, with $\mathbb{E}\left[\left|\mathbf{d}_{s, k}^{l}(p)\right|^{2}\right]=P_{s}$. The data symbols are drawn from a $M$-QAM constellation. The precoder $\mathbf{V}_{s, k}^{l} \in$ $\mathbb{C}^{N_{t} \times S_{s}}, \forall k \in\{1, \ldots, K\}$ is unitary; that is, $\left(\mathbf{V}_{s, k}^{l}\right)^{H} \mathbf{V}_{s, k}^{l}=\mathbf{I}_{S_{s}}$.

\section{Precoders and Equalizers Design}

In this section, we design the IA precoders and derive new iterative frequency domain equalizers by taking into account both the ICI and residual intertier interference. Due to the symmetry of the scenario considered, we will only describe the design of the IA precoders at the small-cell UTs and the iterative equalizer for the macro BS.

3.1. Signal Quantization. To compute the small-cell $k$ precoder $\mathbf{V}_{s, k}^{l}$ the knowledge of the cross-tier channel $\mathbf{H}_{m s, k}^{l}$ is required. In the following, we assume that this information is made available to the corresponding small-cell after being quantized:

$$
\widehat{\mathbf{H}}_{m s, k}^{l}=f_{\mathrm{Q}}\left(\operatorname{Re}\left\{\mathbf{H}_{m s, k}^{l}\right\}\right)+j f_{\mathrm{Q}}\left(\operatorname{Im}\left\{\mathbf{H}_{m s, k}^{l}\right\}\right),
$$

where $f_{Q}(\cdot)$ denotes a quantization function and the operators $\operatorname{Re}\{\cdot\}$ and $\operatorname{Im}\{\cdot\}$ denote the real and imaginary part of $\mathbf{H}_{m s, k}^{l}$. In the next sections we will consider only uniform quantizers with $2^{B}$ levels equally spaced between the clipping levels $-A_{m}$ and $A_{m}$. The quantization of a matrix is performed element wise.

3.2. Precoders Design. To design the small-cell UT precoders we use IA and enforce that all interference is contained within the column space of matrix $\mathbf{A}_{s}^{l}$. The interference subspace matrix may be optimized to maximize some system metric. For simplicity, we consider this matrix randomly selected accordingly to a given probability distribution, known by all terminals. The $\mathrm{UT}_{s, k}$ precoder can be mathematically described by

$$
\mathbf{V}_{s, k}^{l}=\operatorname{null}\left(\mathbf{Q}_{s}^{l} \mathbf{H}_{m s, k}^{l}\right),
$$

where $\mathbf{Q}_{s}^{l}=\operatorname{null}\left(\mathbf{A}_{s}^{l}\right)$ denotes the orthogonal complement of the subspace defined by matrix $\mathbf{A}_{s}^{l}$. The use of a random matrix reduces the need for information exchange between the two tiers.

3.3. Iterative Equalizer Design for the Macro-Cell. The equalizer must deal with both the ICI and the intertier interference. Linear equalizers have poor performance for SC-FDMA 


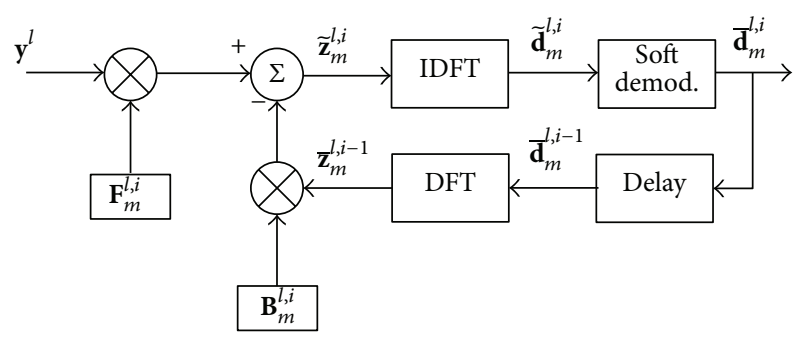

FIGURE 2: Iterative macrocell receiver structure based on IB-DFE principle.

based systems, due to the residual ICI, and consequently they are not well suited also for heterogeneous systems. Consequently, we consider instead an iterative nonlinear equalizer, the IB-DFE, to tackle at the same time both the ICI intertier and the interference.

In Figure 2 we present the proposed IB-DFE receiver structure, for the $i$ th iteration. The received signal on the $l$ th subcarrier and $i$ th iteration, before the IDFT operation, is

$$
\widetilde{\mathbf{x}}_{m}^{l, i}=\mathbf{F}_{m}^{l, i} \mathbf{y}_{m}^{l}-\mathbf{B}_{m}^{l, i} \mathbf{\mathbf { x }}_{m}^{l, i-1}
$$

where $\overline{\mathbf{x}}_{m}^{l, i}=\left[\overline{\mathbf{x}}_{m}^{l, i}(1), \ldots, \overline{\mathbf{x}}_{m}^{l, i}(p), \ldots, \overline{\mathbf{x}}_{m}^{l, i}\left(S_{m} K_{m}\right)\right]^{T} . \mathbf{F}_{m}^{l, i} \in$ $\mathbb{C}^{S_{m} K_{m} \times M_{r}}$ denotes the feedforward matrix and $\mathbf{B}_{m}^{l, i} \in$ $\mathbb{C}^{S_{m} K_{m} \times S_{m} K_{m}}$ the feedback matrix. The sequence $\left\{\overline{\mathbf{x}}_{m}^{l, i}(p)\right\}_{l=1}^{L}$ is the IDFT of the detector output $\left\{\overline{\mathbf{d}}_{m}^{l, i}(p)\right\}_{l=1}^{L}$. For normalized QPSK constellations (i.e., $\overline{\mathbf{d}}_{m}^{l, i}(p)= \pm 1 \pm j$ ) the average values are given by (see [23] for details),

$$
\overrightarrow{\mathbf{d}_{m}^{l, i}}(p)=\tanh \left(\frac{\mathbf{L}_{m}^{(l, i) \operatorname{Re}}(p)}{2}\right)+j \tanh \left(\frac{\mathbf{L}_{m}^{(l, i) \operatorname{Im}}(p)}{2}\right),
$$

where

$$
\begin{aligned}
\mathbf{L}_{m}^{(l, i) \operatorname{Re}}(p) & =\frac{2}{\sigma_{m}^{(l, i)^{2}}} \operatorname{Re}\left\{\mathbf{d}_{m}^{l, i}(p)\right\}, \\
\mathbf{L}_{m}^{(l, i) \operatorname{Im}}(p) & =\frac{2}{\sigma_{m}^{(l, i)^{2}}} \operatorname{Im}\left\{\mathbf{d}_{m}^{l, i}(p)\right\}, \\
\sigma_{m}^{(l, i)^{2}} & =\frac{1}{2 L} \sum_{l^{\prime}=0}^{L-1}\left|\widehat{\mathbf{d}}_{m}^{l^{\prime, i}}(p)-\overline{\mathbf{d}}_{m}^{l^{\prime}, i}(p)\right|^{2} .
\end{aligned}
$$

The extension to other constellations is considered in [24]. The hard decision $\widehat{\mathbf{d}}_{m}^{l, i}(p)$ associated with data symbol $\mathbf{d}_{m}^{l}(p)$ is

$$
\begin{aligned}
\widehat{\mathbf{d}}_{m}^{l, i}(p)= & \operatorname{sign}\left(\operatorname{Re}\left\{\tilde{\mathbf{d}}_{m}^{l, i}(p)\right\}\right) \\
& +j \operatorname{sign}\left(\operatorname{Im}\left\{\tilde{\mathbf{d}}_{m}^{l, i}(p)\right\}\right) .
\end{aligned}
$$

It can be shown that $\overline{\mathbf{x}}_{m}^{l, i} \approx \Psi_{m}^{i^{2}} \overline{\mathbf{x}}_{m}^{l}+\Psi_{m}^{i} \mathbf{e}_{m}^{l}$, where $\mathbf{e}_{m}^{l}$ is a zero mean error vector and $\Psi_{m}^{i}$ a diagonal correlation matrix.
The correlation coefficient computed for the $j$ th $L$-length data block is

$$
\Psi_{m}^{i}(p)=\frac{\mathbb{E}\left[\hat{\mathbf{d}}_{m}^{l, i-1}(p) \mathbf{d}_{m}^{l^{*}}(p)\right]}{\mathbb{E}\left[\left|\mathbf{d}_{m}^{l}(p)\right|^{2}\right]}, \quad l=1, \ldots, L .
$$

This scalar $\Psi_{m}^{i}(p)$ represents a measure of the reliability of the estimates of the $p$ th $L$-length data block associated with the $i$ th iteration, which can be approximately given by

$$
\Psi_{m}^{i}(p) \approx \frac{1}{2 L} \sum_{l=0}^{L-1}\left|\Psi_{m}^{(l, i) \operatorname{Re}}(p)+\Psi_{m}^{(l, i) \operatorname{Im}}(p)\right|^{2}
$$

where

$$
\begin{aligned}
& \Psi_{m}^{(l, i) \operatorname{Re}}(p)=\tanh \left(\frac{\left|\mathbf{L}_{m}^{(l, i) \operatorname{Re}}(p)\right|}{2}\right), \\
& \Psi_{m}^{(l, i) \operatorname{Im}}(p)=\tanh \left(\frac{\left|\mathbf{L}_{m}^{(l, i) \operatorname{Im}}(p)\right|}{2}\right) .
\end{aligned}
$$

For a given iteration, we have seen that the iterative nonlinear equalizer is characterized by the matrices $\mathbf{F}_{m}^{l, i}$ and $\mathbf{B}_{m}^{l, i}$, which are selected to minimize the average bit error rate (BER). For a QPSK constellation with Gray mapping the BER is approximately given by [23]

$$
\mathrm{BER}^{l} \approx Q\left(P_{m}\left(\frac{1}{L} \sum_{l=1}^{L} \mathrm{MSE}^{l, i}\right)^{-1}\right),
$$

where $Q(x)$ denotes the $Q$ function and $\mathrm{MSE}_{l}$ denotes the mean square error on the frequency domain samples $\widetilde{\mathbf{z}}_{m}^{l}$, which is given by

$$
\begin{aligned}
\operatorname{MSE}^{l, i} & =\mathbb{E}\left[\left\|\widetilde{\mathbf{z}}_{m}^{l, i}-\mathbf{z}_{m}^{l}\right\|^{2}\right] \\
& =\mathbb{E}\left[\operatorname{tr}\left(\left(\widetilde{\mathbf{z}}_{m}^{l, i}-\mathbf{z}_{m}^{l}\right)\left(\widetilde{\mathbf{z}}_{m}^{l, i}-\mathbf{z}_{m}^{l}\right)^{H}\right)\right] .
\end{aligned}
$$

After some mathematical manipulations, it can be shown that (15) can be reduced to

$$
\begin{aligned}
\operatorname{MSE}^{l, i}= & \operatorname{tr}\left(\mathbf{F}_{m}^{l, i} \mathbf{R}_{\mathbf{y}_{m}^{l}}^{l} \mathbf{F}_{m}^{(l, i)^{H}}\right)+\operatorname{tr}\left(\mathbf{B}_{m}^{l, i} \mathbf{R}_{\overline{\mathbf{z}}_{m}, \overline{\mathbf{z}}_{m}}^{i-1} \mathbf{B}_{m}^{(l, i)^{H}}\right) \\
& +S_{m} K_{m}-2 \operatorname{tr}\left(\operatorname{Re}\left\{\mathbf{F}_{m}^{l, i} \mathbf{R}_{\mathbf{y}_{m}^{l}, \mathbf{z}_{m}}^{l}\right\}\right) \\
& +2 \operatorname{tr}\left(\operatorname{Re}\left\{\mathbf{B}_{m}^{l, i} \mathbf{R}_{\overline{\mathbf{z}}_{m}, \mathbf{z}_{m}}^{i-1}\right\}\right) \\
& -2 \operatorname{tr}\left(\operatorname{Re}\left\{\mathbf{B}_{m}^{l, i, i} \mathbf{R}_{\mathbf{z}_{m}, \mathbf{y}_{m}^{l}}^{l, i-1} \mathbf{F}_{m}^{(l, i,)^{H}}\right\}\right) .
\end{aligned}
$$


The different correlation matrices of (16) are given by

$$
\begin{aligned}
\mathbf{R}_{\mathbf{y}_{m}^{l}}^{l} & =\mathbb{E}\left[\mathbf{y}_{m}^{l^{*}} \mathbf{y}_{m}^{l^{T}}\right] \\
& =\mathbf{H}_{m m}^{l} \mathbf{R}_{m} \mathbf{H}_{m m}^{l^{H}}+K_{s} S_{s} \mathbf{A}_{s}^{l} \mathbf{A}_{s}^{l^{H}}+\mathbf{R}_{n}, \\
\mathbf{R}_{\overline{\mathbf{z}}_{m}, \overline{\mathbf{z}}_{m}}^{i-1} & =\mathbb{E}\left[\overline{\mathbf{z}}_{m}^{*} \overline{\mathbf{z}}_{m}^{T}\right]=\Psi_{m}^{(i-1)^{2}} \mathbf{R}_{m}, \\
\mathbf{R}_{\overline{\mathbf{z}}_{m}, \mathbf{z}_{m}}^{i-1} & =\mathbb{E}\left[\overline{\mathbf{z}}_{m}^{*} \mathbf{z}_{m}\right]=\Psi_{m}^{(i-1)^{2}} \mathbf{R}_{m}, \\
\mathbf{R}_{\mathbf{y}_{m}^{l}, \mathbf{z}_{m}}^{l} & =\mathbb{E}\left[\mathbf{y}_{m}^{l^{*}} \mathbf{z}_{m}\right]=\mathbf{H}_{m m}^{l} \mathbf{R}_{m}, \\
\mathbf{R}_{\mathbf{z}_{m}, \mathbf{y}_{m}^{l}}^{l, i-1} & =\mathbb{E}\left[\overline{\mathbf{z}}_{m}^{*} \mathbf{y}_{m}^{l}\right]=\Psi_{m}^{(i-1)^{2}} \mathbf{R}_{m} \mathbf{H}_{m m}^{l^{H}}
\end{aligned}
$$

with $\mathbf{R}_{m}=P_{m} \mathbf{I}_{S_{m} K_{m}}$ and $\mathbf{R}_{n}=\left(\sigma_{\text {noise }}^{2} / P_{m}+\sigma_{Q}^{2}\right) \mathbf{I}_{M_{r}}$ being the correlation matrices of the data symbols and of the residual noise, respectively. $\sigma_{Q}^{2}$ denotes the power of the noise introduced by the quantization procedure, which is assumed approximately Gaussian distributed.

To optimize the parameters of the iterative equalizer, that is, matrices $\mathbf{F}_{m}^{l, i}$ and $\mathbf{B}_{m}^{l, i}$, we use as a metric the average BER (14). This optimization problem may be mathematically formulated as follows:

$$
\begin{aligned}
\min _{\mathbf{F}_{m}^{l, i}, \mathbf{B}_{m}^{l, i}} & \mathrm{MSE}^{l, i} \\
\text { s.t } & \frac{1}{L} \sum_{l=1}^{L} \operatorname{tr}\left(\mathbf{F}_{m}^{l, i} \mathbf{H}_{m m}^{l}\right)=S_{m} K_{m} .
\end{aligned}
$$

The Lagrangian and Karush-Kuhn-Tucker (KKT) conditions $[27,28]$ of optimization problem (18) are, respectively,

$$
\begin{aligned}
& \mathbb{L}\left(\mathbf{F}_{m}^{l, i}, \mathbf{B}_{m}^{l, i}, \mu_{m}\right) \\
& \quad=\mathrm{MSE}^{l, i}-\mu_{m}\left(\frac{1}{L} \sum_{l}^{L} \operatorname{tr}\left(\mathbf{F}_{m}^{l, i} \mathbf{H}_{m m}^{l}\right)-S_{m} K_{m}\right), \\
& \nabla_{\mathbf{F}_{m}^{l, i}} \mathbb{E}\left(\mathbf{F}_{m}^{l, i}, \mathbf{B}_{m}^{l, i}, \mu_{m}\right)=0, \\
& \nabla_{\mathbf{B}_{m}^{l, i}} \mathbb{L}\left(\mathbf{F}_{m}^{l, i}, \mathbf{B}_{m}^{l, i}, \mu_{m}\right)=0, \\
& \frac{1}{L} \sum_{l=1}^{L} \operatorname{tr}\left(\mathbf{F}_{m}^{l, i} \mathbf{H}_{m m}^{l}\right)-S_{m} K_{m}=0,
\end{aligned}
$$

where $\mu_{m}$ is the Lagrangian multiplier. The solution to optimization problem (18) is

$$
\begin{aligned}
\mathbf{F}_{m}^{l, i} & =\boldsymbol{\Omega}_{m}^{i} \mathbf{H}_{m m}^{l^{H}}\left(\mathbf{A}_{m}^{l}\right)^{-1}, \\
\mathbf{B}_{m}^{l, i} & =\mathbf{F}_{m}^{l, i} \mathbf{H}_{m m}^{l}-\mathbf{I}_{S_{m} K_{m}}, \\
\mathbf{\Omega}_{m}^{i} & =\left(\mathbf{I}_{M_{0}}-\Psi_{m}^{(i-1)^{2}}\right)-\mu_{m} \mathbf{I}_{M_{0}},
\end{aligned}
$$

where $\mathbf{A}_{m}^{l}=\mathbf{H}_{m m}^{l}\left(\mathbf{I}_{S_{m} K_{m}}-\boldsymbol{\Psi}_{m}^{(i-1)^{2}}\right) \mathbf{H}_{m m}^{l^{H}}+K_{s} S_{s} \mathbf{A}_{s}^{l} \mathbf{A}_{s}^{l^{H}}+$ $\left(\sigma_{\text {noise }}^{2} / P_{m}+\sigma_{\mathrm{Q}}^{2}\right) \mathbf{I}, \mu_{m}$ is the Lagrangian multiplier, and $\sigma_{\mathrm{Q}}^{2}$ is the quantization noise power. For the first iteration $(i=1)$ matrix $\Psi_{m}^{0}$ and vector $\overline{\mathbf{x}}_{m}^{l, 0}$ are a null matrix and a null vector, respectively.

As discussed above, the macrocell UTs precoders and the iterative equalizer used at the CU can be easily obtained by following the same procedure used to compute the small-cell UTs precoders and the macrocell BS equalizer, respectively.

The semianalytical approach consists in using (16) and (17) to obtain an estimate of the average BER; see (14). Relatively to the complexity, each iteration of the proposed receiver structures is similar to the linear MMSE based equalizer, since the most part of complexity comes from the matrix inversion to compute the feedforward coefficients. Therefore, the additional complexity of the proposed receiver is basically given by the number of equalizer iterations.

\section{Performance Results}

In this section we access the performance of the proposed multitier IA-precoding and IB-DFE techniques. The number of antennas at the macro-BS, macro-UTs, small-cell APs, and small-cell UTs is set to $M_{r}=4, M_{t}=3, N_{r}=3$, and $N_{t}=3$, respectively. Each user terminal, both macro- and small-cell UTs, transmits one stream; that is, $S_{m}=S_{s}=1$. The number of macrocell UTs and the number of small-cells are set to $K_{m}=K_{s}=2$. The block and FFT size are set both to $L=128$ and QPSK with Gray mapping was adopted. The channels between each transmitter and receiver pair are uncorrelated and severely time-dispersive, each one with rich multipath propagation and uncorrelated Rayleigh fading for different multipath components. Specifically, we assume $L_{p}=$ 32 symbol spaced paths with uniform power delay profile and uncorrelated Rayleigh fading. The same conclusions could be drawn for other multipath fading channels, if the number of separable multipath components is high. In addition, we assume perfect CSI at the receiver side (the cross-tier channels are quantized) and synchronization. We use as a metric for performance evaluation the average bit error rate (BER) as a function of $E_{b} / N_{0}$, with $E_{b}$ denoting the average bit energy and $N_{0}$ denoting the one-sided noise power spectral density. For the sake of comparisons we also include the MFB.

Let us start by considering the case without cross-tier quantization $(B=\infty)$. In Figures 3 and 4 we present results for the macrocell and small-cells (i.e., at the CU), respectively. In these two figures, we present results for the first, second, and fourth iterations of the proposed iterative equalizer. The MFB curve is also plotted as a baseline. For the first iteration the IB-DFE reduces to the linear MMSE based equalizer, since $\Psi_{m}^{0}$ and $\overline{\mathbf{x}}_{m}^{l, 0}$ are a null matrix and a null vector, respectively. As the iteration number increases the performance of the proposed method improves. For the 4 th iteration and for high SNR a performance close to MFB is reached. This indicates that the proposed multitier IA in conjunction with IB-DFE efficiently removes the generated ICI and intertier interferences. Moreover, the semianalytical approximation is quite precise. Indeed, for the first iteration, where the IB-DFE based equalizer reduces to a linear MMSE based frequency domain equalizer, they almost overlap. This is a consequence of the accuracy of the Gaussian assumption made to derive the 


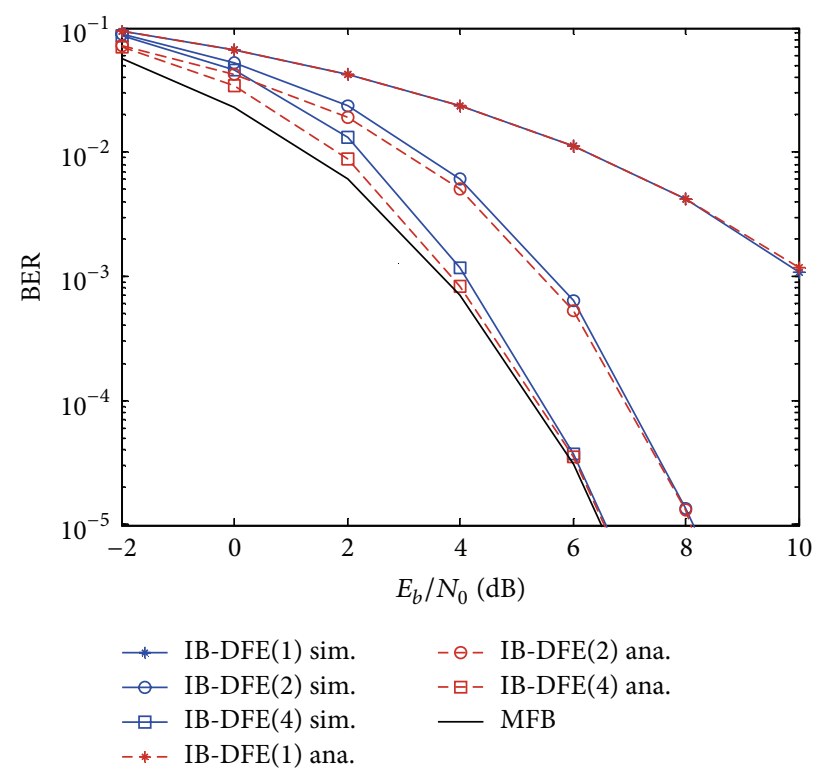

FIgURE 3: Performance evaluation at the macrocell, no quantization.

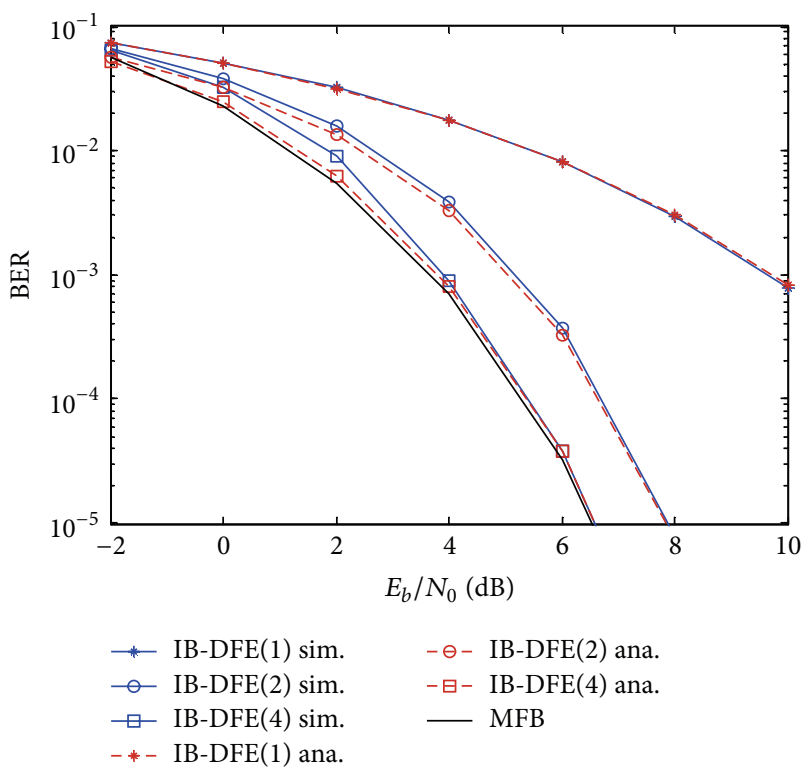

FIGURE 4: Performance evaluation at the central unit, no quantization.

proposed semianalytical results. For highly time-dispersive channels with rich multipath propagation the inter-symbolinterference (ISI) is high and therefore the signals of interest may be assumed Gaussian. Nevertheless, at the second and fourth iterations the ISI is already significantly reduced. Therefore, the Gaussian approximation of the residual ISI is less accurate, mainly for low $E_{b} / N_{0}$ values.

Now let us consider the case with quantization. The results for this case are presented in Figures 5 and 6 for macrocell and central unit, respectively. For this case, due to the quantization procedure there is residual interference between the UTs' signals that may affect significantly the system performance. From these figures, we can verify that

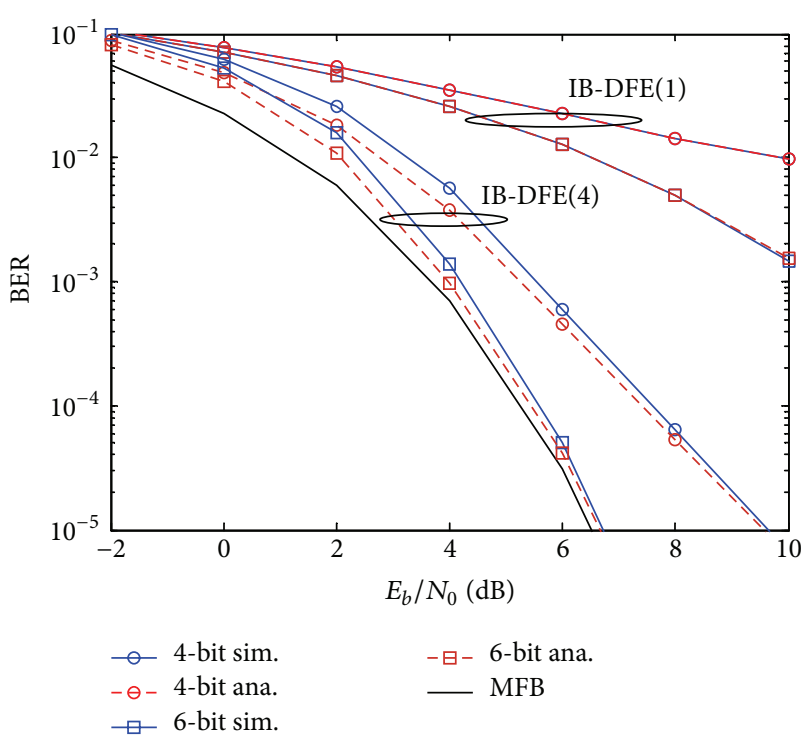

FIGURE 5: Performance evaluation at the macrocell, with quantization.

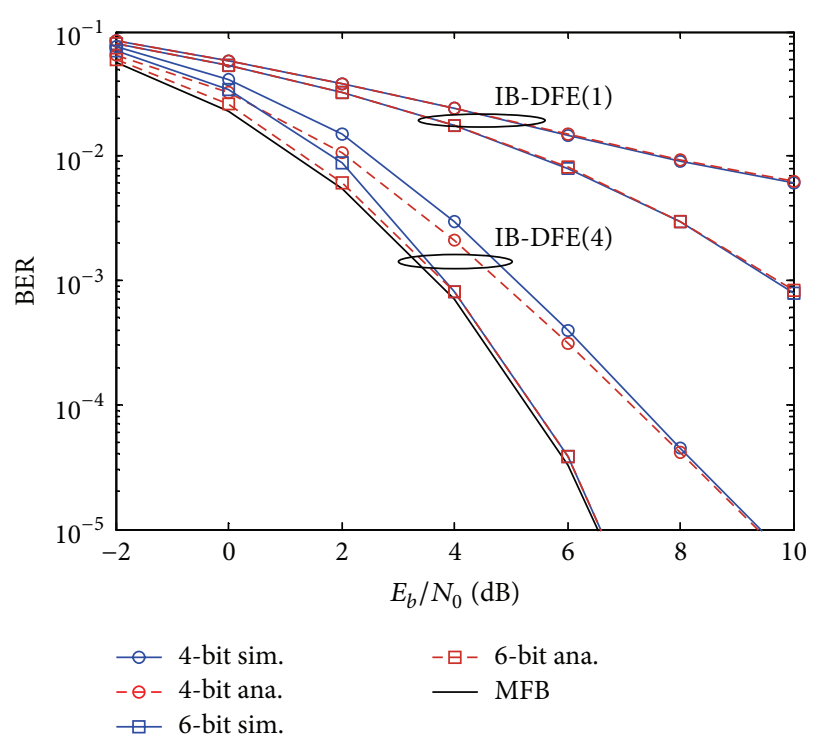

FIgUre 6: Performance evaluation at the central unit, with quantization.

the semianalytic results are still close to the ones obtained by simulation. Furthermore, with just four iterations and 6 bits we achieve close to the MFB performance. We also can see that when we have a single iteration there is a significant performance degradation from the 4-bit to the 6-bit performance since the interuser interference increases as the number of quantization bits decreases. For the 4 th iteration the performance degradation from the 4 bit to the 6 bit curve is lower, which means that increasing the number of iterations of the equalizer reduces considerably the impact inherent to the noise introduction by the quantization. Furthermore, with just four iterations and 6 bits we achieve close to the MFB performance and we verify that as the 
number of quantization bits increases the performance of the proposed method improves. With just 6 bits the achieved performance is very close to the unquantized approach and for the fourth iteration it is even very close to the MFB.

By comparing the results at the small-cells to the ones at the macro we verify that the performance of the macro is slightly degraded in comparison to the small-cells. This fact stems from the additional degrees of freedom available at the $\mathrm{CU}$, in comparison with the macro-BS. The macro-BS has 3 antennas and the CU has 4 antennas, 2 from each aggregated AP.

\section{Conclusions}

In this paper, we proposed the use of IA in conjunction with iterative space-frequency equalization to enable the coexistence of a set of small-cells within the coverage area of a macrocell. A frequency reuse of one has been considered. By using IA the intertier/system interference is aligned at a predefined subspace, at both cells. Nevertheless, due to the availability of only imperfect channel knowledge at the user terminals the alignment is poor and this results in severe interference. To deal with this type of interference we proposed to adapt the IB-DFE from the single-tier case to the multitier scenario. To achieve this the optimization of the iterative equalizer was done by taking into account this additional interference. We also proposed an accurate semianalytical approach for obtaining the performance of the proposed schemes. The results showed that IA-precoding works well with iterative equalization allowing a close-tooptimum space-diversity gain; that is, for the considered scenario 4 iterations were sufficient to achieve close to MFB performance. Therefore, the proposed equalizers are robust to the intertier interference and well suited to deal with the intertier interference limitations of the heterogeneous networks.

\section{Conflict of Interests}

The authors declare that there is no conflict of interests regarding the publication of this paper.

\section{Acknowledgments}

This work was supported by the Portuguese Fundação para a Ciência e Tecnologia (FCT) COPWIN (PTDC/EEITEL/1417/2012), ADIN (PTDC/EEI-TEL/2990/2012), and HETCOP (UID/EEA/50008/2013) projects. The work of D. Castanheira was supported by FCT under a postdoctoral grant SFRH/BPD/95375/2013.

\section{References}

[1] J. Hoadley and P. Maveddat, "Enabling small cell deployment with HetNet," IEEE Wireless Communications, vol. 19, no. 2, pp. 4-5, 2012.

[2] Z. Becvar and P. Mach, "Mitigation of redundant handovers to femtocells by estimation of throughput gain," Mobile Information Systems, vol. 9, no. 4, pp. 315-330, 2013.
[3] A. Goldsmith, S. A. Jafar, I. Maric, and S. Srinivasa, "Breaking spectrum gridlock with cognitive radios: an information theoretic perspective," Proceedings of the IEEE, vol. 97, no. 5, pp. 894-914, 2009.

[4] V. R. Cadambe and S. A. Jafar, "Interference alignment and degrees of freedom of the K-user interference channel," IEEE Transactions on Information Theory, vol. 54, no. 8, pp. 34253441, 2008.

[5] M. A. Maddah-Ali, A. S. Motahari, and A. K. Khandani, "Communication over MIMO X channels: interference alignment, decomposition, and performance analysis," IEEE Transactions on Information Theory, vol. 54, no. 8, pp. 3457-3470, 2008.

[6] K. Gomadam, V. R. Cadambe, and S. A. Jafar, "A distributed numerical approach to interference alignment and applications to wireless interference networks," IEEE Transactions on Information Theory, vol. 57, no. 6, pp. 3309-3322, 2011.

[7] S. W. Peters and R. W. Heath Jr., "Cooperative algorithms for MIMO interference channels," IEEE Transactions on Vehicular Technology, vol. 60, no. 1, pp. 206-218, 2011.

[8] D. Kim and M. Torlak, "Optimization of interference alignment beamforming vectors," IEEE Journal on Selected Areas in Communications, vol. 28, no. 9, pp. 1425-1434, 2010.

[9] W. Shin, W. Noh, K. Jang, and H.-H. Choi, "Hierarchical interference alignment for downlink heterogeneous networks," IEEE Transactions on Wireless Communications, vol. 11, no. 12, pp. 4549-4559, 2012.

[10] S. K. Sharma, S. Chatzinotas, and B. Ottersten, "Interference alignment for spectral coexistence of heterogeneous networks," EURASIP Journal on Wireless Communications and Networking, vol. 2013, no. 1, article 46, 2013.

[11] J. Assunção, D. Castanheira, F. Oliveira, A. Silva, and A. Gameiro, "IA-precoding with IB-DFE scheme for uplink SCFDMA heterogeneous networks," in Proceedings of the International Conference on Wireless and Mobile Communication Systems (WMCS '14), Lisbon, Portugal, October-November 2014.

[12] D. Castanheira, A. Silva, and A. Gameiro, "Set optimization for efficient interference alignment in heterogeneous networks," IEEE Transactions on Wireless Communications, vol. 13, no. 10, pp. 5648-5660, 2014.

[13] H. Holma and A. Toskala, LTE for UMTS-OFDMA and SCFDMA Based Radio Access, John Wiley \& Sons, 2009.

[14] H. G. Myung and D. J. Goodman, Single Carrier FDMA: A New Air Interface for Long Term Evolution, John Wiley \& Sons, Chichester, UK, 2008.

[15] D. Falconer, S. L. Ariyavisitakul, A. Benyamin-Seeyar, and B. Eidson, "Frequency domain equalization for single-carrier broadband wireless systems," IEEE Communications Magazine, vol. 40, no. 4, pp. 58-66, 2002.

[16] A. Gusmão, R. Dinis, J. Conceição, and N. Esteves, "Comparison of two modulation choices for broadband wireless communications," in Proceedings of the 51st IEEE Vehicular Technology Conference Proceedings (VTC-Spring '00), vol. 2, pp. 1300-1305, IEEE, Tokyo, Japan, May 2000.

[17] N. Benvenuto, R. Dinis, D. Falconer, and S. Tomasin, "Single carrier modulation with nonlinear frequency domain equalization: an idea whose time has come-again," Proceedings of the IEEE, vol. 98, no. 1, pp. 69-96, 2010.

[18] N. Benvenuto and S. Tomasin, "Block iterative DFE for single carrier modulation," Electronics Letters, vol. 38, no. 19, pp. 1144$1145,2002$. 
[19] N. Benvenuto and S. Tomasin, "Iterative design and detection of a DFE in the frequency domain," IEEE Transactions on Communications, vol. 53, no. 11, pp. 1867-1875, 2005.

[20] R. Dinis, P. Silva, and T. Araújo, "Turbo equalization with cancelation of nonlinear distortion for CP-assisted and zero-padded MC-CDM schemes," IEEE Transactions on Communications, vol. 57, no. 8, pp. 2185-2189, 2009.

[21] M. Luzio, R. Dinis, and P. Montezuma, "SC-FDE for offset modulations: an efficient transmission technique for broadband wireless systems," IEEE Transactions on Communications, vol. 60, no. 7, pp. 1851-1861, 2012.

[22] A. Silva, S. Teodoro, R. Dinis, and A. Gameiro, "Iterative frequency-domain detection for IA-precoded MC-CDMA systems," IEEE Transactions on Communications, vol. 62, no. 4, pp. 1240-1248, 2014.

[23] R. Dinis, P. Silva, and A. Gusmão, "IB-DFE receivers with space diversity for CP-assisted DS-CDMA and MC-CDMA systems," European Transactions on Telecommunications, vol. 18, no. 7, pp. 791-802, 2007.

[24] J. Silva, R. Dinis, N. Souto, and P. Montezuma, "Single-carrier frequency domain equalisation with hierarchical constellations: an efficient transmission technique for broadcast and multicast systems," IET Communications, vol. 6, no. 13, pp. 2065-2073, 2012.

[25] A. Gusmão, P. Torres, R. Dinis, and N. Esteves, "A turbo FDE technique for reduced-CP SC-based block transmission systems," IEEE Transactions on Communications, vol. 55, no. 1 , pp. 16-20, 2007.

[26] H. Nam and Y. Kim, "A low-complexity single-carrier frequency-division multiple access transmitter," International Journal of Communication Systems, vol. 25, no. 11, pp. 1489-1495, 2012.

[27] S. Boyd and V. Lieven, Convex Optimization, Cambridge University Press, Cambridge, UK, 2009.

[28] R. T. Rockafellar, Convex Analysis, vol. 28 of Princeton Landmarks in Mathematics and Physics, Princeton University Press, 1997. 

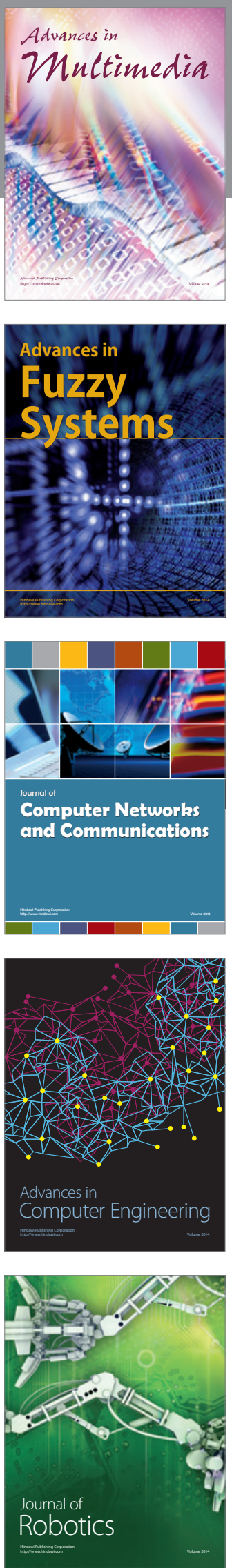

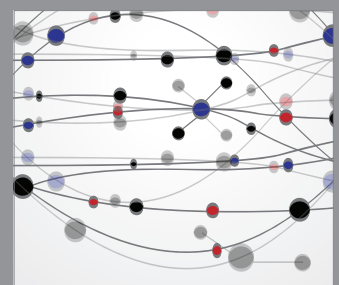

The Scientific World Journal
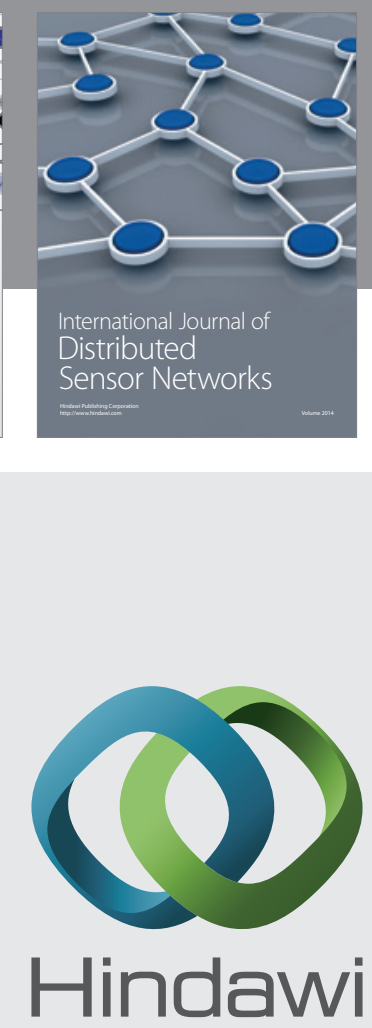

Submit your manuscripts at

http://www.hindawi.com
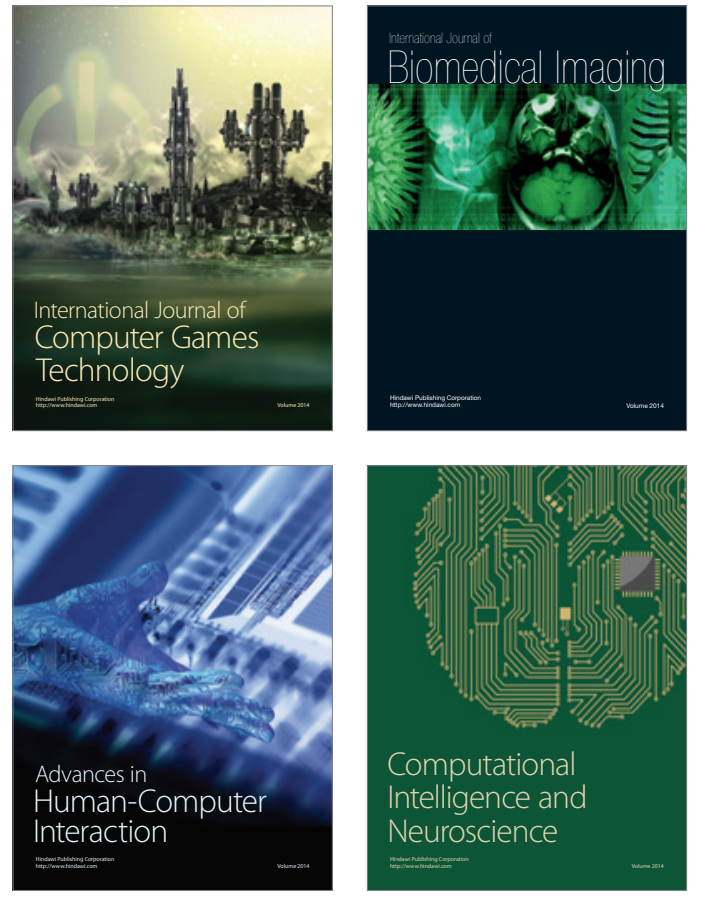
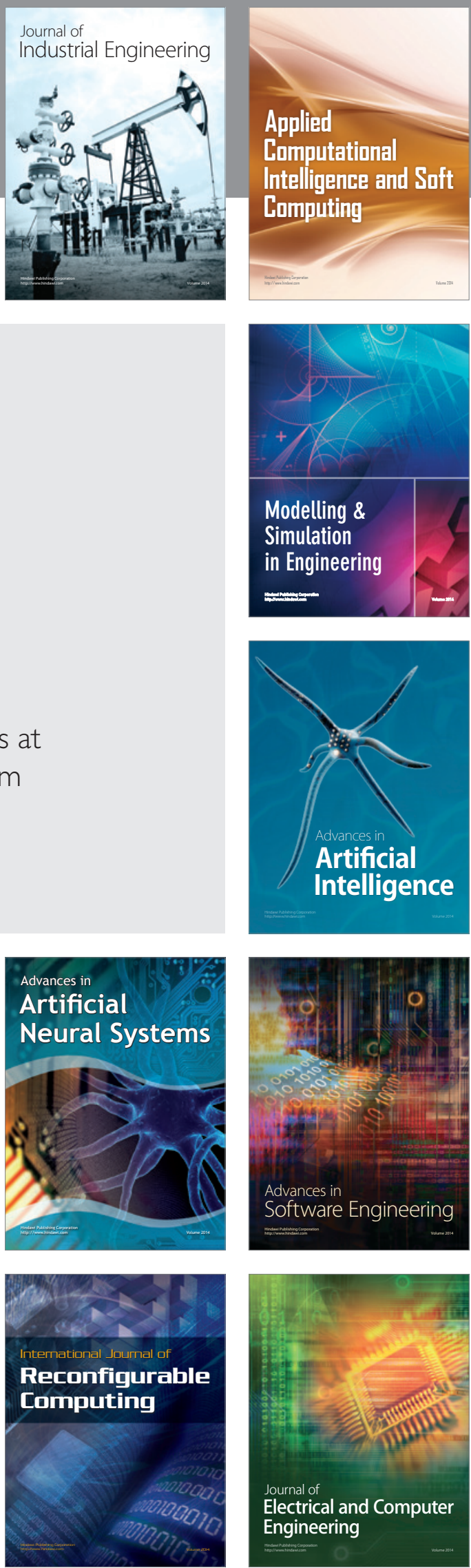